\title{
ELLIPTIC MULTIPARAMETER EIGENVALUE PROBLEMS
}

\author{
by P. A. BINDING and K. SEDDIGHI
}

(Received 13th August 1985)

\section{Preliminaries}

We study the eigenproblem

$$
W_{m}(\lambda) x_{m}=0 \neq x_{m} \in H_{m}, \quad m=1, \ldots, k
$$

where

$$
W_{m}(\lambda)=T_{m}-\sum_{n=1}^{k} \lambda_{n} V_{m n}
$$

and $T_{m}, V_{m n}$ are self-adjoint operators on separable Hilbert spaces $H_{m}$. We assume the $T_{m}$ to be bounded below with compact resolvents, and the $V_{m n}$ to be bounded and to satisfy an "ellipticity" condition. If $k=1$ then ellipticity is automatic, and if each $T_{m}$ is positive definite then the problem is "left definite".

We show that certain eigenvectors $x_{m}$ of $\left(^{*}\right)$ corresponding to $\lambda \in \mathbb{R}^{k}$ generate a basis $X$ of decomposable tensors for a subspace $G$ of $H=\bigotimes_{m=1}^{k} H_{m}$ of finite codimension. More specifically, we define $k$ (in general indefinite) inner products, relative to which (i) $X$ is positive and orthogonal and (ii) $G$ has a common orthocomplement $F$ in $H$. Bounds for $\operatorname{dim} F$ are given, and in particular if each $T_{m}$ is nonnegative definite then $\operatorname{dim} F \leqq 2 \prod_{n=1}^{k} \operatorname{dim} N\left(T_{m}\right)$.

\section{Introduction}

Completeness and expansion theorems have formed a central part of multiparameter spectral theory since early in this century. In particular, Hilbert's work [14; Chapter 21] on a special pair of linked Sturm-Liouville equations has been successively generalised to what is nowadays called the "left-definite" (LD) case of the eigenproblem (*) of Section 0 . Such problems include a number of systems of ordinary differential equations arising from separation of variables of classical boundary value problems into various coordinate systems-cf. [1; Chapter 1]. Indeed, early work on multiparameter theory was generally motivated by such problems, in the Sturm-Liouville form

$$
\left(p_{m} y_{m}^{\prime}\right)^{\prime}+q_{m} y_{m}=\sum_{j=1}^{k} \lambda_{n} r_{m n} y_{m},
$$


for eigenfunctions $y_{m}$ in suitable subspaces of $L_{2}\left[a_{m}, b_{m}\right]$. We remark that an alternative "right definite" condition can also be used, but the present expansion theory [21] cannot handle all such applications.

In the abstract framework used here, Källström and Sleeman have given a completeness theorem for the LD case-see [19: Chapter 5]. LD is the combination of "ellipticity" of the $V_{m n}$ and the "definiteness" of the $T_{m}$. Ellipticity is automatic when $k=1$, and involves two definiteness conditions on combinations of the $V_{m n}$ when $k=2$. The latter are automatically satisfied, for example, for the equations (1.1) whenever they arise from the plane Helmholtz equation in any of the four coordinate systems permitting separation of variables. The other, "definiteness", condition can be a drawback of $L D$, since effectively it requires positive definiteness of each $T_{m}$-for more on this question, see Example 5.5 at the end. In the (elliptic) differential equation case, it is frequently the case that the $T_{m}$ are essentially positive definite (e.g. for (1.2), $p_{m}$ takes one sign) but the "lower order" terms and boundary conditions give $T_{m}$ a finite number $v\left(T_{m}\right)$ of negative eigenvalues.

Even in the one parameter case

$$
T x=\lambda V x
$$

(where we drop subscripts for simplicity and where LD simply means positive definiteness of $T$ ), the results need modification when $v(T)>0$. In particular, complex eigenvalues may occur, and the eigenvectors need not be dense in $H$, so root vectors may be required for completeness. In the finite dimensional case, such results can be found in Weierstrass's 1880's theory of canonical forms of pencils (see [17], $\$ 108$ or [12], Chapter 1.3 for modern accounts in the context of self-adjoint operators on indefinite inner product spaces). In the Sturm-Liouville case, Richardson was aware (apparently as a by-product of his 1910's work on two-parameter problems) of the need for complex eigenvalues, and explicit examples requiring complex eigenvalues and/or root vectors can be found in recent work of Fleckinger and Mingarelli-cf. [10]. The abstract setting, which includes both the above cases, was investigated by Pontryagin in the 1940's in the context of indefinite inner product spaces, and can be found in ([7], Chapter IX). An infinite dimensional completeness result involving compact operators was apparently treated first by Iohvidov in 1950, but without a published proof. We recently proved a completeness result for (1.2) [6], and we shall use it in a fundamental way in Section 3. Roughly, the LD result holds on a subspace $G$ of codimension at most $3 v(T)$, and the known finite dimensional result holds on a suitable complement of $G$. We remark that one can also approach (1.2) via Langer's "spectral function", but this is somewhat involved-see e.g. Daho and Langer [8] for the Sturm-Liouville case. Incidentally, all the above references treat invertible $T$.

Various generalisations of $(1.2)$ can be treated via reduction to $\left(^{*}\right)$ (cf. Greenlee [13] and Roach and Sleeman [18] who use $k=1$ and $k=2$ respectively to treat quadratic eigenvalue problems). The only investigation we know of under ellipticity alone seems to be by Faierman [9] for the Sturm-Liouville case (1.1) with $k=2$. We are grateful to Dr. Faierman for his preprints which have enabled us to give the following comparison between his work and ours. Roughly, [9] takes advantage of a function space setting to derive uniform convergence of double Fourier-type series, but at the cost of continuity 
conditions which can be checked only after rather detailed analysis of an elliptic partial differential equation

$$
\Delta_{n} x=\lambda_{n} \Delta_{0} x
$$

for $n=2$. Our work uses $k$ abstract equations (1.3) on $\bigotimes_{m=1}^{k} H_{m}$ for $n=1 \ldots k$ to derive completeness results in abstract (but strong) metrics under weak continuity conditionsin fact $L_{\infty}$ coefficients would suffice for (1.1). Faierman attacks (1.3), with $\Delta_{n}$ possibly noninvertible, using an a posteriori nondegeneracy condition ([9], Assumption 6.1). We use a more direct nondegeneracy condition, which we call "regularity", permitting transformation to a case where $\Delta_{n}$ is invertible for each $n=1 \ldots k$. Incidentally, regularity holds automatically if $\Delta_{0}$ is $1-1$, and the latter can be checked on the original data either in the finite dimensional case ([2], Theorem 8.5.1) or in the Sturm-Liouville case (1.1) when $\Delta_{0}$ is $1-1 \Leftrightarrow \operatorname{det}\left[r_{m n}\right]$ vanishes on a ( $k$ dimensional Lebesgue) null set. While Faierman's nondegeneracy condition is removable (as he has informed us), he expands only functions with a common compact support, $\Omega$ say, on which $\operatorname{det}\left[r_{m n}\right] \neq 0$. It seems, then, that one could use a version of (1.3) where $\Delta_{0}$ is $1-1$, defined via quadratic forms restricted to $\Omega$.

Our plan is as follows. In Section 2 we state the assumptions, including definitions of ellipticity and regularity, and we show how to transform the eigenvalues so that each operator $\Delta_{n}$ is self-adjoint and bounded below with compact inverse. In particular, $v\left(\Delta_{n}\right)$ is finite, and (1.3) is equivalent to

$$
B_{n} x=\lambda_{n}^{-1} x,
$$

where $B_{n}=\Delta_{n}^{-1} \Delta_{0}$ is compact. In Section 3 we prove that the $B_{n}$ commute, and that bases for joint eigenspaces of the $B_{n}$ can be constructed out of the eigenvectors of $\left({ }^{*}\right)$. In Section 4 we combine these results with those of [6] to give a general completeness theorem. Roughly, the LD result holds on a subspace $G \subseteq H$ of codimension at most $3 \sum_{n=1}^{k} v\left(\Delta_{n}\right)$, and on a suitable complement of $G$ the problem reduces to one studied by Atkinson ([2], Chapter 6). We also show how to bound $\operatorname{codim} G$ in terms of the original data $\left({ }^{*}\right)$. Finally in Section 5 we discuss the situation when each $T_{m}$ is nonnegative definite-in this case the bound on $\operatorname{codim} G$ improves to $2 v$ where $v=\prod_{n=1}^{k} \operatorname{dim} N\left(T_{m}\right)$. In particular, if $v=1$ (e.g. in the Sturm-Liouville case (1.1) with separated end conditions) then $\operatorname{codim} G=1$ or 2 , and we characterize both cases directly in terms of the original data.

\section{Transformations of the problem}

Of the self-adjoint operators on a Hilbert space $H$, we shall distinguish by $B S(H)$ those which are bounded, by $P I(H)$ those which have a bounded positive definite inverse, and by $C R(H)$ those which are bounded below with compact resolvent. Necessarily

$$
T \in C R(H) \Rightarrow T+\zeta I \in P I(H) \Rightarrow(T+\zeta I)^{-1} \in B S(H)
$$

for large positive $\zeta$. Recall the standing

Assumption 2.1. $T_{m} \in C R\left(H_{m}\right), V_{m n} \in B S\left(H_{m}\right), 1 \leqq m, n \leqq k$. 
From now on, $H$ will denote the (Hilbert) tensor product $\bigotimes_{m=1}^{k} H_{m}$. We start by inducing $V_{m n}$ into $H$, yielding an operator $V_{m n}^{\dagger} \in B S(H)$, and we note that $V_{m n}^{\dagger}$ and $V_{j l}^{\dagger}$ commute if $m \neq j$. Thus we can define $\Delta_{0}$ as the $k \times k$ determinant $\operatorname{det}\left[V_{m n}^{\dagger}\right]$, with $\Delta_{0 m n}$ as the corresponding $(m, n)$ th cofactor. If $u=u_{1} \otimes \cdots \otimes u_{k}$ is a decomposable tensor with $\left\|u_{m}\right\|=1, \quad m=1 \ldots k$, then the expression $\delta_{0 m n}(u)=\left(u, \Delta_{0 m n} u\right)$ is a $(k-1) \times(k-1)$ determinant with elements of the form $\left(u_{j}, V_{j l} u_{j}\right)$. The original definition of "ellipticity" [15] requires $k$ linear combinations of the $\delta_{0 m n}(u)$ to be positively bounded below, but ([4], p. 480) shows that this positivity may be assumed of each $\delta_{0 m n}(u)$, after a nonsingular linear transformation of the eigenvalues $\lambda$. Then ([4], Theorem 3.1) shows that we may assume "ellipticity" in the form:

Assumption 2.2. $\Delta_{0 m n} \in P I(H), 1 \leqq m, n \leqq k$.

(If $k=1$ then we write $\Delta_{0 m n}=I$, so ellipticity is automatic). We emphasize that the original assumption is on the original data $\left({ }^{*}\right)$.

We induce $T_{m}$ similarly into $H$ as a self-adjoint operator $T_{m}^{\dagger}$. Let $D$ denote the intersection $\bigcap_{m=1}^{k} D\left(T_{m}^{\dagger}\right)$, which is easily seen to be dense in $H$. For $n=1 \ldots k, \Delta_{n}$ is then defined as the closure in $H$ of

$$
\left.\Delta_{n}\right|_{D}=\sum_{m=1}^{k} \Delta_{0 m n} T_{m}^{\dagger}
$$

Using the fact that $\Delta_{0 m n}$ and $T_{m}^{\dagger}$ commute on $D\left(T_{m}^{\dagger}\right)$, one easily shows that $\left.\Delta_{n}\right|_{D}$ is symmetric, and it will turn out that $\Delta_{n}$ is self-adjoint.

We are now in a position to state our "regularity" condition:

Assumption 2.3. For $n=1 \ldots k, \Delta_{0} y=0 \neq y \in H$ implies

$$
\left(y, \Delta_{n} x_{n}\right) \neq 0 \text { for some } x_{n} \in N\left(\Delta_{0}\right) \cap D\left(\Delta_{n}\right) .
$$

This is satisfied vacuously if $\Delta_{0}$ is $1-1$. First we shall discuss the consequence for (1.2), i.e. for $k=1$. Then $\Delta_{0}=V$ and $\Delta_{1}=T$, and the following result shows that "regularity" gives the usual definition ([11], p. 25) for pencils in finite dimensions.

Theorem 2.4 If $k=1$ then there is an open real interval $J=]-\rho, \rho[$ such that $T+\varepsilon V$ is $1-1$ for all nonzero $\varepsilon \in J$.

Proof. For each $\zeta \in \mathbb{C}$, write $T(\zeta)=T+\zeta V$. If no interval $J$ exists as stated, then

$$
0 \in \sigma_{p}\left(T+\zeta_{j} V\right) \quad j=1,2, \ldots
$$

for a sequence of real $\zeta_{j} \rightarrow 0$ as $j \rightarrow \infty$. From the properties of self-adjoint holomorphic families $([16]$, p. 386) we conclude that the eigenvalues of $T(\zeta)$ are holomorphic at $\zeta=0$, and therefore $\left.0 \in c^{\prime}, T(\zeta)\right)$ for all small $|\zeta|$. In particular, we can find $y^{\varepsilon} \in H$ of unit norm such that

$$
(T+\varepsilon i V) y^{\varepsilon}=0 \text { for all small real } \varepsilon
$$


Elementary manipulations now give

$$
\left(y^{\varepsilon}, V y^{\eta}\right)=\left(y^{\varepsilon}, T y^{\eta}\right)=0 \text { for all small nonzero real } \varepsilon \text { and } \eta \text {. }
$$

In particular, if $S$ is the linear span of such $y^{\varepsilon}$, then

$$
(y, T y)=0 \text { for all } y \in S .
$$

It follows from ([7], Lemma IX.1.1) that $\operatorname{dim} S$ is finite, so let $y_{l}=y^{\varepsilon_{l}}, l=1 \ldots m$, be a basis for $S$. Suppose

$$
y_{n}=\sum_{l=1}^{m} \alpha_{l} y_{l}
$$

where $\varepsilon_{n} \neq \varepsilon_{l}, l=1 \ldots m$. Then

$$
\begin{aligned}
\varepsilon_{n} V y_{n}=i T y_{n} & =i \sum_{l=1}^{m} \alpha_{l} T y_{l} \\
& =\sum_{l=1}^{m} \varepsilon_{l} \alpha_{l} V y_{l} .
\end{aligned}
$$

It follows from (2.2) that $V y=0$, where

$$
y=\sum_{l=1}^{m}\left(\varepsilon_{n}-\varepsilon_{l}\right) \alpha_{l} y_{l}
$$

Now for any $x \in N(V) \cap D(T)$, we have

$$
\left(y_{l}, T x\right)=\left(-\varepsilon_{l} i V y_{l}, x\right)=\left(-\varepsilon_{l} i y_{l}, V x\right)=0
$$

so it follows from (2.3) that $(y, T x)=0$. Thus $y=0$ by Assumption 2.3, and so each $\alpha_{t}=0$ by (2.3). But then $y_{n}=0$ by (2.2) and we contradict $\left\|y_{n}\right\|=1$.

We now proceed to general $k$, and we shall repeatedly use a construction based on translations of $\sigma\left(T_{m}\right)$. To motivate this, we note that

$$
T_{m}+\alpha I_{m} \in P I\left(H_{m}\right) \quad \text { for large positive } \alpha \text {. }
$$

Hence although $T_{m}^{\dagger} \notin C R(H)$ in general, it is true that $T_{m}^{\dagger}+\zeta I \in P I(H)$ for large positive $\zeta$. Thus if $T_{m}$ is replaced by $T_{m}+\zeta I_{m}$, then the analogue of $\Delta_{n}$, which we denote by $\Delta_{n}(\zeta)$, belongs to $P I(H)$. Specifically, we take $\Delta_{n}(\zeta)$ as the closure of $\sum_{m=1}^{k} \Delta_{0 m n}\left(T_{m}^{\dagger}+\zeta I\right)$, and so

$$
\Delta_{n}(\zeta)=\Delta_{n}+\zeta \Sigma_{n}, \quad \Sigma_{n}=\sum_{m=1}^{k} \Delta_{0 m m}, \quad n=1 \ldots k
$$


Theorem 2.5. After a possible translation of the eigenvalues, we may assume, in addition to Assumptions $2.1-3$, that $\Delta_{n}$ is $1-1$ and belongs to $C R(H)$, for $n=1 \ldots k$.

Proof. Choose large enough positive $\alpha$ to satisfy (2.4). Throughout this proof, $n$ will denote an integer between 1 and $k$. Since $D\left(T_{m}+\alpha I_{m}\right)=D\left(T_{m}\right),\left.\Delta_{n}(\alpha)\right|_{D}$ is essentially selfadjoint by ([20], Lemma 4.4). Since $\Sigma_{n} \in B S(H), \Delta_{n}(\alpha)$ is self-adjoint, so $\Delta_{n}(\alpha) \in C R(H)$ by ([4], Theorem 3.1). Thus $\Delta_{n}=\Delta_{n}(\alpha)-\alpha \Sigma_{n} \in C R(H)$ by ([5], Lemma 1).

If we replace $\lambda$ by $\lambda+\varepsilon$ where $\varepsilon \in \mathbb{R}^{k}$, then we must replace $T_{m}$ by $W_{m}(\varepsilon)$ in $\left(^{*}\right)$. Thus $\Delta_{n}$ is replaced by the closure of

$$
\sum_{m=1}^{k} \Delta_{0 m n} W_{m}(\varepsilon)^{\dagger}=\left.\Delta_{n}\right|_{D}+\varepsilon_{n} \Delta_{0}
$$

i.e. by $\Delta_{n}+\varepsilon_{n} \Delta_{0}$. Here we use standard identities ([15], equation 2.2)) and $\Delta_{0} \in B S(H)$.

Since $\Delta_{n} \in C R(H)$, we may apply Theorem 2.4 , with $(T, V)$ replaced by $\left(\Delta_{n}, \Delta_{0}\right)$, to conclude that $\Delta_{n}+\varepsilon_{n} \Delta_{0}$ is $1-1$ for all translations with $\varepsilon_{n}$ small and nonzero. Moreover $\Delta_{n}+\varepsilon_{n} \Delta_{0} \in C R(H)$ by $([5]$, Lemma 1$)$, and if $\Delta_{0} y=0 \neq y$ then

$$
\left(y,\left(\Delta_{n}+\varepsilon_{n} \Delta_{0}\right) x\right)=\left(y, \Delta_{n} x\right) \neq 0 \text { for some } x \in N\left(\Delta_{0}\right) \cap D\left(\Delta_{n}+\varepsilon_{n} \Delta_{0}\right)
$$

Thus Assumption 2.3 remains valid, and the other assumptions are unaffected by the translation.

\section{The operators $B_{n}$}

By Theorem 2.5, we may define compact operators

$$
B_{n}=\Delta_{n}^{-1} \Delta_{0}, \quad n=1, \ldots, k
$$

and as in [4], they will be the main tool for the completeness theorem when $k>1$. In this section we shall extend two crucial properties of the $B_{n}$ from the LD case, by means of analytic perturbation theory. The first property is as follows.

Theorem 3.1. The $B_{n}$ are pairwise commutative.

Proof. Using (2.5) for arbitrary $\zeta \in \mathbb{C}$, we define $R_{n}$ as the resolvent set for

$$
E_{n}=-\Sigma_{n}^{-1 / 2} \Delta_{n} \Sigma_{n}^{-1 / 2}
$$

and we claim that $\zeta \in R_{n}$ if and only if $\Delta_{n}(\zeta)$ is $1-1$. Indeed

$$
\begin{array}{r}
\Delta_{n}(\zeta) x=0 \neq x \in H \\
\Leftrightarrow \quad\left(\Delta_{n}+\zeta \Sigma_{n}\right) x=0 \neq x \\
\Leftrightarrow E_{n} y=\zeta y, \quad y=\Sigma_{n}^{1 / 2} x \neq 0 .
\end{array}
$$

Since both $\Delta_{n}(\zeta)$ and $E_{n}$ have purely point spectrum, our cfaim is established. 
Choose large enough $\alpha>0$ to satisfy (2.4). Then $\Delta_{n}(\alpha)$ is positive definite, so by Theorem $2.5,\{0, \alpha\} \subset R_{n}$. Since $E_{n}$ is self-adjoint, we find

$$
Z_{\varepsilon}=\{\zeta:|\zeta|<\varepsilon\} \cup\{\zeta:|\zeta-\alpha|<\varepsilon\} \cup\{\zeta: \alpha-2 \varepsilon<|2 \zeta-\alpha|<\alpha+2 \varepsilon, \operatorname{Im} \zeta>0\}
$$

is a connected and simply connected open subset of $R_{n}$ for small enough $\varepsilon>0$.

Define $B_{n}(\zeta)=\Delta_{n}(\zeta)^{-1} \Delta_{0}$ for $\zeta \in R_{n}$. By $([4]$, Theorem 4.2),

$$
B_{m}(\zeta) B_{n}(\zeta)=B_{n}(\zeta) B_{m}(\zeta)
$$

for each $\zeta$ belonging to the real interval $] \alpha, \alpha+\varepsilon\left[\subset R_{n}\right.$. It follows that the holomorphic operator valued functions on both sides of (3.2) agree for all $\zeta \in Z_{\varepsilon}$ and in particular for $\zeta=0 \multimap c f$. ([16], pp. 367-368).

The second property we need expresses a connection between common eigenvectors of the $B_{n}$ and eigentensors $x^{\otimes}=x_{1} \otimes \cdots \otimes x_{k}$ where $x_{m}$ satisfy $\left(^{*}\right)$ for $\lambda \in \mathbb{R}^{k}$. The set of $x$ satisfying $B_{n} x=\mu_{n} x, n=1 \ldots k$, is denoted by $E(\mu)$ whenever $\mu \in \mathbb{R}^{k}$ and the resulting subspace is nontrivial. We define $k+1$ sesquilinear forms on $E(\mu)$ by $(x, y)_{l}=\left(x, \Delta_{l} y\right)$, $l=0 \ldots k$.

Theorem 3.2. Any eigentensor of $\left({ }^{*}\right)$, with $\lambda \in \mathbb{R}^{k}$, belongs to $E(\mu)$ where $\mu_{n}=\lambda_{n}^{-1}$, $n=1 \ldots k$. Conversely if $\mathbf{0} \neq \mu \in \mathbb{R}^{k}$ and, for some $n,(x, x)_{n}$ is of one sign for all nonzero $x \in E(\mu)$, then $E(\mu)$ has a (finite) basis of eigentensors which are $(,)_{l}$-orthogonal for each $l=0 . . k$.

Proof. The first sentence is standard-cf. ([4], Theorem 6.1). The converse is proved via the technique of Theorem 3.1. Note that $\mu_{n} \neq 0$ for each $n=1 \ldots k$. By ([4], p. 485), we have the identity

$$
\prod_{l=1}^{k} \lambda_{l} B_{l}(\zeta)=\left(T_{m}^{\dagger}+\zeta I\right)^{-1} \sum_{n=1}^{k} V_{m n}^{\dagger} \lambda_{n} \prod_{l=1, l \neq n}^{k} \lambda_{l} B_{l}(\zeta)
$$

for $\lambda \in \mathbb{C}^{k}$ and $\left.\zeta \in\right] \alpha, \alpha+\varepsilon[$, the notation being as in (3.1) and (3.2).

Rearranging, we obtain

$$
\left(I+(\zeta-\alpha)\left(T_{m}^{\dagger}+\alpha I\right)^{-1}\right) \prod_{l=1}^{k} \lambda_{l} B_{l}(\zeta)=\left(T_{m}^{\dagger}+\alpha I\right)^{-1} \sum_{n=1}^{k} V_{m n}^{\dagger} \lambda_{n} \prod_{l=1, l \neq n}^{k} \lambda_{l} B_{l}(\zeta)
$$

Since the homomorphic operator-valued functions on both sides of (3.3) agree for all $\zeta \in] \alpha, \alpha+\varepsilon\left[\right.$, and hence for all $\zeta \in Z_{\varepsilon}$ (3.1), we may set $\zeta=0$, apply both sides to

$$
x=\lambda_{n} B_{n} x \quad \text { where } \quad \lambda_{n}=\mu_{n}^{-1}, \quad n=1 \ldots k,
$$

and rearrange to give

$$
W_{m}(\lambda)^{\dagger} x=0, \quad m=1 \ldots k
$$


Let $P_{m}$ be the orthoprojector of $H_{m}$ onto $N\left(W_{m}(\lambda)\right)$. Replacing $T_{m}, V_{m n}$ by $P_{m} T_{m}, P_{m} V_{m n}$ restricted to $N\left(W_{m}(\lambda)\right)$, we obtain a finite dimensional version of $\left({ }^{*}\right)$ with one eigenvalue, viz. $\lambda$. The replacement for $H$ is just $E(\mu)$, and so the conclusion follows directly from ([2], Theorem 7.9.1).

\section{Completeness in the general case}

Although our results lead to fundamental sets of vectors in $H$, it is more convenient to express them in terms of complete orthogonal bases of vectors in the subspaces $D_{n}=D\left(\left|\Delta_{n}\right|^{1 / 2}\right), n=1 \ldots k$. We shall endow $D_{n}$ with two sesquilinear forms,

$$
(x, y)_{n}=\left(x, \Delta_{n} y\right) \text { and }[x, y]_{n}=\left(x,\left|\Delta_{n}\right| y\right)
$$

for $y \in D\left(\Delta_{n}\right)$, with completion for $y \in D_{n}$. For more on these constructions, see e.g. ([6], $\S 3)$. In particular $D_{n}$ is a Hilbert space under $[,]_{n}$, and topological concepts (e.g. closure) will be understood in the $[,]_{n}$ sense. Algebraic concepts (e.g. orthogonality) will however be understood in the $(,)_{n}$ sense. Note that the number $v_{n}$ of negative eigenvalues of $\Delta_{n}$ is finite by Theorem 2.5 , so $D_{n}$ is a Pontryagin space under $(,)_{n}$.

We shall give a preliminary completeness result for $n$ fixed between 1 and $k$. If $\mu$ is an eigenvalue of $B_{n}$ then we write $N_{n}^{j}(\mu)=N\left(B_{n}-\mu I\right)^{j}$. The case $j=1$ corresponds to the eigenspace $N\left(B_{n}-\mu I\right)$, which we denote by $E_{n}(\mu)$. Given $j \geqq 2 v_{n}+1$, it can be shown ([7], p. 191) that $N_{n}^{j}(\mu)=N_{n}^{j+1}(\mu)$, and we write

$$
F_{n}(\mu)=N_{n}^{j}(\mu)+N_{n}^{j}(\bar{\mu})
$$

for such $j$.

Lemma 4.1. $D_{n}=N\left(\Delta_{0}\right) \oplus F_{n} \oplus G_{n}$ where $N\left(\Delta_{0}\right)=F_{n}(0), F_{n}$ is an $(,)_{n}$-orthogonal direct sum of the $F_{n}(\mu)$ and $\operatorname{dim} F_{n} \leqq 3 v_{n} . G_{n}$ is the closed linear span of the remaining $F_{n}(\mu)$ and is a Hilbert space under $(,)_{n}=[.]_{n}$. The summands in $G_{n}$ are $(,)_{n}$-orthogonal and satisfy $F_{n}(\mu)=E_{n}(\mu)$ and $\mu \in \mathbb{R}$ in each case.

Proof. The results follow from ([6], Corollaries 4 and 5). In particular, $F_{n}(0)=E_{n}(0)=$ $N\left(\Delta_{0}\right)$ follows from $([6]$, Corollary 1$)$.

We now remove some of the dependence on $n$ by means of the constructions

$$
E(\mu)=\bigcap_{n=1}^{k} E_{n}\left(\mu_{n}\right) \text { and } F(\mu)=\bigcap_{n=1}^{k} F_{n}\left(\mu_{n}\right)
$$

for $\mu_{n} \in \sigma_{p}\left(B_{n}\right), n=1 \ldots k$. It will turn out that $E(\mu)$ is needed only when $\mathbf{0} \neq \boldsymbol{\mu} \in \mathbb{R}^{k}$, as in Theorem 3.2.

Lemma 4.2. For each $n=1 \ldots k, D_{n}=N\left(\Delta_{0}\right) \oplus F \oplus G$, where $N\left(\Delta_{0}\right)=F(0), F$ is a finite direct sum of the $F(\mu)$, and $G$ is the closed linear span of the remaining $F(\mu)$, for each of which $\mathbf{0} \neq \mu \in \mathbb{R}^{k}$ and $F(\mu)=E(\mu)$. 
Remark. It is an easy calculation to show that the $[,]_{n}$ are topologically equivalent, so $G$ does not depend on ${ }_{n}$.

Proof. Set $j \geqq 2 v_{n}+1$ for each $n=1 \ldots k$. If $x \in F_{n}\left(\mu_{n}\right)$ then $x=y+z$ where $y \in N_{n}^{j}\left(\mu_{n}\right)$ and $z \in N_{n}^{j}\left(\bar{\mu}_{n}\right)$. By Theorem 3.1,

$$
B_{m} x=B_{m} y+B_{m} z \in N_{n}^{j}\left(\mu_{n}\right)+N_{n}^{j}\left(\bar{\mu}_{n}\right)=F_{n}\left(\mu_{n}\right)
$$

so $F_{n}\left(\mu_{n}\right)$ is $B_{m}$-invariant for each $m=1 \ldots k$. We now decompose the summands of Lemma 4.1 into root subspaces of $B_{m}$ for each $m \neq n$ in turn. Evidently $N\left(\Delta_{0}\right)=F_{n}(0)$ remains unchanged, and $D_{n}$ is decomposed into a direct sum of the $F(\mu)-\mathrm{cf}$. ([2], Theorem 3.6.1).

We claim that $F(\mu)=E(\mu)$ and $\mu \in \mathbb{R}^{k}$ hold for all but finitely many $\mu$. Indeed if we take a Jordan basis for any $B_{m}$ in any finite sum of the $F(\mu)$ then the Jordan chain lengths are prescribed by Lemma 4.1 with $n$ replaced by $m$. In particular, all but finitely many chains have length one and correspond to real eigenvalues.

The main completeness result is a refinement of the above.

Theorem 4.3. The direct sums in Lemma 4.2 are (, ), -orthogonal for each $l=0 \ldots k$, as are the summands in $G$ for different $\mu$. Moreover $F$ can be chosen so that $G$ is a Hilbert space under $(,)_{n}=[,]_{n}$ for each $n=1 \ldots k$, and a set of eigentensors for $\left({ }^{*}\right)$ exists forming a complete orthogonal basis of $G$ in all of these inner products.

Proof. Suppose $x \in N\left(\Delta_{0}\right)$ and $y \in F(\mu), \mathbf{0} \neq \boldsymbol{\mu} \in \mathbb{C}^{k}$. Then $(x, y)_{0}=0$ is trivial, and $\mu_{n} \neq 0$ implies $(x, y)_{n}=0$ by ([7], Theorem II.2.5). Now suppose $u \in E(\boldsymbol{\mu}), \mathbf{0} \neq \boldsymbol{\mu} \in \mathbb{R}^{k}$ and $v \in F(\rho)$, $\rho \neq \mu$. If $\rho_{n} \neq \mu_{n}$ then $(u, v)_{n}=0$, again by ([7], Theorem II.2.5). Thus for $l=1 \ldots k$

$$
(u, v)_{l}=\left(\Delta_{l} u, v\right)=\mu_{l}^{-1}\left(\Delta_{0} u, v\right)=\mu_{n} \mu_{l}^{-1}\left(\Delta_{n} u, v\right)=\mu_{n} \mu_{l}^{-1}(u, v)_{n}
$$

must vanish. The claims of the first sentence are now established.

If $(,)_{n} \neq[,]_{n}$ for some $n$ for infinitely many $E(\mu)$, then we contradict Lemma 4.1. Thus $F$ can be chosen to include all $E(\mu)$ for which $(,)_{n} \neq[,]_{n}$ for some $n$, and so $(,)_{n}=[,]_{n}$ on $G$ for all $n$ by the orthogonality already established. Theorem 3.2 now completes the proof.

We shall now discuss some questions related to the dimension of $F$. One way of reducing $\operatorname{dim} F$ is to include in $G$ those $E(\mu)$ for which $(,)_{n}$ is definite for some $n$ (and hence for all $n$ by (4.1)). The price is that $G$ need no longer be a Hilbert space, although it will be a Pontryagin space, under each $(,)_{n}$. Another way of reducing $\operatorname{dim} F$ is to remove subspaces of $E(\mu)$ from certain $F(\mu) \neq E(\mu)$. More precisely, we may decompose $F(\mu)$ into $L_{n}(\mu)=F_{n}(\mu) \cap F_{n}$ and $M_{n}(\mu)=F_{n}(\mu) \cap G_{n} \subseteq E_{n}\left(\mu_{n}\right)$ for $\mu \neq 0$ by Lemma 4.1. Let $M(\mu)=\bigcap_{n=1}^{k} M_{n}(\mu)$ and let $L(\mu)$ be the $(,)_{l}$-orthogomplement of $M(\mu)$ in $F(\mu)$ for some $l$ (and hence for all $l=1 \ldots k$ by (4.1)). By the rules of dimension (cf. ([17], p. 51)),

$$
\operatorname{dim} L(\mu)=\operatorname{dim} F(\mu)-\operatorname{dim} M(\mu) \leqq \sum_{n=1}^{k} \operatorname{dim} L_{n}(\mu)
$$


We now sum the $L(\mu)$ and $M(\mu)$ over those $\mu$ for which $F(\mu) \subseteq F$, yielding subspaces $L$ and $M$ of $F$. By Lemma 4.1, $(,)_{n}=[,]_{n}$ on $M$, and by (4.2) and Lemma 4.1,

$$
\operatorname{dim} L \leqq \sum_{n=1}^{k} 3 v_{n}
$$

Thus $\operatorname{dim} F$ can be reduced to $\operatorname{dim} L$, and $G$ remains a Hilbert space in each inner product $(,)_{n}, n=1 \ldots k$. The price is that we cannot assert the existence of a decomposable basis for $M$.

Finally let us consider bounds for $\operatorname{dim} F$ in terms of the original data $\left({ }^{*}\right)$. Since the various possibilities for $\operatorname{dim} F$ (e.g. (4.3)) can all be expressed in terms of the $v_{n}$, it will suffice to estimate the latter. For simplicity we shall bound $v_{1}$ in the case $k=2$-the general case is analogous but involves extra terms. By Assumptions 2.1-2, we have

$$
\Delta_{1}=T_{1}^{\dagger} S_{1}+T_{2}^{\dagger} S_{2}, \quad H=\left(H_{1}^{-} \oplus H_{1}^{+}\right) \oplus\left(H_{2}^{-} \oplus H_{2}^{+}\right)
$$

where $S_{2}=V_{12}^{\dagger}$ and $S_{1}=-V_{22}^{\dagger} \in S P(H)$ and $H_{m}^{+}, H_{m}^{-}$are the positive and negative spectral subspace of $T_{m}$. In particular, $\operatorname{dim} H_{m}^{-}=v\left(T_{m}\right)<\infty$. We seek an upper bound for the dimension of a $(,)_{1}$-negative subspace in $H_{1}^{-} \otimes H_{2}^{+}$. Suppose $T_{1} \geqq \alpha I_{1}, V_{12} \geqq \beta I_{1}$ and $V_{22} \geqq \gamma I_{2}$ with $\alpha, \gamma<0$ and $\beta>0$. Let $T_{2}$ possess $\delta$ eigenvalues (repeated according to multiplicity) in the interval $[0, \alpha \gamma / \beta]$. Any subspace $K$ of $H_{1}^{-} \otimes H_{2}^{+}$of dimension $>\delta v\left(T_{1}\right)$ must contain an element $x$ satisfying

$$
\left(x, T_{2} x\right)>\alpha \gamma(x, x) / \beta
$$

Thus $S_{2}^{-1 / 2} K$ contains $y=S_{2}^{-1 / 2} x$ satisfying

$$
\left(y, T_{2}^{\dagger} S_{2} y\right)>\alpha \gamma(y, y) \geqq\left(y, T_{1}^{\dagger} S_{1} y\right)
$$

i.e. $(y, y)_{1}>0$. Since $S_{2}$ is an isomorphism, we have $\delta v\left(T_{1}\right)$ as our required bound. A similar bound (say $\varepsilon v\left(T_{2}\right)$ ) can be found for the maximal dimension of $(,)_{1}$-negative subspaces of $H_{1}^{+} \otimes H_{2}^{-}$, and so our final bound for $v_{1}$ is

$$
\left(v\left(T_{1}\right)+\varepsilon\right)\left(v\left(T_{2}\right)+\delta\right)-\varepsilon \delta
$$

\section{The nonnegative definite case}

Throughout this section we shall employ:

Assumption 5.1. $T_{m}$ is nonnegative definite for each $m=1 \ldots k$,

in addition to Assumptions 2.1-3. It is convenient to discuss the one parameter case (1.2) first. By Theorem 2.5 we may replace $\lambda$ by $\lambda+\varepsilon$, where $\varepsilon \in \mathbb{R}$ is small, so that $T$ is replaced by an invertible operator $T^{\prime}=T+\varepsilon V$. Then we may define a compact operator $B^{\prime}=\left(T^{\prime}\right)^{-1} V$ and associated subspaces $E^{\prime}(\mu)$ and $F^{\prime}(\mu)$ for $\mu \in \sigma_{p}\left(B^{\prime}\right)$, as in the prelude to Lemma 4.1. It is convenient to write $\eta=\varepsilon^{-1}$. 


\section{Lemma 5.2.}

(i) The eigenvalues $\lambda$ of (1.2) and $\mu$ of $B^{\prime}$ are all real.

(ii) $F^{\prime}(\mu)=E^{\prime}(\mu)$ for all $\mu \neq \eta$.

(iii) $E^{\prime}(\eta)=N\left(B^{\prime}-\eta I\right)=N(T)$ and $\left.F^{\prime}(\eta)=N\left(B^{\prime}-\eta I\right)^{2}\right)$.

Proof. (i) $B^{\prime} x=\mu x$ is equivalent to

$$
\mu T x=(1-\varepsilon \mu) V x
$$

so if $\mu=0$ then $\mu \in \mathbb{R}$ and $x \in N(V)$. From now on we assume $\mu \neq 0$. Then (5.1) shows that (1.2) is satisfied with $\lambda=\mu^{-1}-\varepsilon$. Conversely, (1.2) implies

$$
x=(\lambda+\varepsilon) B^{\prime} x
$$

so if $x \neq 0$ then again we have $\mu=(\lambda+\varepsilon)^{-1} \neq 0$, i.e. $\lambda=\mu^{-1}-\varepsilon$.

If $(x, T x) \neq 0$ then $(x, V x) \neq 0$ so $\lambda$ is a ratio of reals, whence $\lambda$ and $\mu$ are both real. If $(x, T x)=0$ then $T x=0$ by Assumption 5.1 so $\mu=\eta$ and $\lambda=0$ are both real.

(ii) It suffices to prove

$$
y \in N\left(\left(B^{\prime}-\mu I\right)^{2}\right) \Rightarrow y \in N\left(B^{\prime}-\mu I\right) .
$$

The left side can be written $\left(B^{\prime}-\mu I\right) y=x$, i.e.

$$
[(1-\varepsilon \mu) V-\mu T] y=T^{\prime} x
$$

where $x$ satisfies $(5.1)$, i.e. $[(1-\varepsilon \mu) V-\mu T] x=0$. Using (5.2) we obtain

$$
0=\left(x, T^{\prime} x\right)
$$

On the other hand, (5.1) can also be written $\mu T^{\prime} x=V x$, so

$$
(x, V x)=\mu\left(x, T^{\prime} x\right)
$$

Using (5.3) twice, we have $(x, V x)=0$ and $(x, T x)=0$ in turn, and so $T x=0$ by Assumption 5.1. As in (i) $x \neq 0$ forces $\mu=\eta$, and so in fact $x=0$, i.e. $y \in N\left(B^{\prime}-\mu I\right)$.

(iii) The first contention is immediate from (5.1). For the second, we shall prove

$$
z \in N\left(\left(B^{\prime}-\eta I\right)^{3}\right) \Rightarrow z \in N\left(\left(B^{\prime}-\eta I\right)^{2}\right) \text {. }
$$

The left side can be written $\left(B^{\prime}-\eta I\right) z=y$, i.e.

$$
-\eta T z=T^{\prime} y
$$

where $y$ and $x$ satisfy (5.2) and (5.1) for $\mu=\eta$, viz.

$$
-\eta T y=T^{\prime} x \text { and } T x=0 .
$$


Thus

$$
0=(x, T z)=-\varepsilon\left(x, T^{\prime} y\right)=(y, T y)
$$

whence $T y=0$. Comparing (5.4) and $T y=0$ with (5.5) we see that $y \in N\left(B^{\prime}-\eta I\right)$ and so indeed $z \in N\left(\left(B^{\prime}-\eta I\right)^{2}\right)$.

By setting $T=\Delta_{n}$ and $V=\Delta_{0}$ we obtain an improvement of Lemma 4.1 that nevertheless still depends on $n$. To remove that dependence we need one more result.

Lemma 5.3. $N\left(\Delta_{n}\right)=N:=\bigotimes_{m=1}^{k} N\left(T_{m}\right)$, for each $n=1 \ldots k$.

Proof. $\left(x, \Delta_{n} x\right)=\sum_{m=1}^{k}\left(x, T_{m}^{\dagger} \Delta_{0 m n} x\right)$ for all $x \in D$ so $\Delta_{n}$ is nonnegative definite. Thus if $x \in N\left(\Delta_{n}\right)$ then there is a sequence $x_{j} \in D$ such that $x_{j} \rightarrow x$ and

$$
\left(x_{j}, \Delta_{n} x_{j}\right) \rightarrow\left(x, \Delta_{n} x\right)=0 \quad \text { as } \quad j \rightarrow \infty .
$$

In particular, the nonnegative sequence

$$
\begin{aligned}
\|\left(\Delta_{0 m n}^{1 / 2}\left(T_{m}^{\dagger}\right)^{1 / 2} x_{j} \|^{2}\right. & =\left(x_{j},\left(\Delta_{0 m n}^{1 / 2}\left(T_{m}^{\dagger}\right)^{1 / 2}\right)^{2} x_{j}\right) \\
& =\left(x_{j}, \Delta_{0 m n} T_{m}^{\dagger} x_{j}\right) \rightarrow 0 \text { as } j \rightarrow \infty
\end{aligned}
$$

Thus $\left(T_{m}^{\dagger}\right)^{1 / 2} x_{j} \rightarrow 0$, and since $\left(T_{m}^{\dagger}\right)^{1 / 2}$ is closed we have $T_{m}^{\dagger} x=0$ for $m=1 \ldots k$. Thus $x \in N-$ see $([4]$, p. 485$)$, and so $N\left(\Delta_{n}\right) \subseteq N$. The reverse implication is obvious.

We are now ready for the main completeness result. It improves Theorem 4.3 sufficiently to make the subsequent remarks in Section 4 unnecessary in this case. Using Theorem 2.5, we translate the eigenvalues $\lambda$ by $\varepsilon$ and we write

$$
\eta_{n}=\varepsilon_{n}^{-1}, \quad B_{n}^{\prime}=\left(\Delta_{n}+\varepsilon_{n} \Delta_{0}\right)^{-1} \Delta_{0}, \quad n=1 \ldots k,
$$

with associated subspaces $F_{n}^{\prime}\left(\mu_{n}\right), E^{\prime}(\mu)$ etc. It is convenient to write $v=\operatorname{dim} N$-see Lemma 5.3.

Theorem 5.4. It is possible to choose $F=F^{\prime}(\eta)$ in Theorem 4.3, with $\operatorname{dim} F \leqq 2 v$.

Proof. By Lemma 5.2, it is possible to choose $F_{n}=F_{n}^{\prime}\left(\eta_{n}\right)$ in Lemma 4.1. Now choose a Jordan basis for $B_{m}^{\prime}, m \neq n$, in the $B_{n}^{\prime}$-invariant subspace $F_{n}^{\prime}\left(\mu_{n}\right)$. Any Jordan chain for $B_{m}^{\prime}$ of length 2 must correspond to eigenvalue $\eta_{m}$ by Lemma 4.1 with $n$ replaced by $m$. By Lemmas 5.2 and 5.3, the first element of such a chain belongs to $N \subset F_{n}^{\prime}\left(\eta_{n}\right)$, and so $F^{\prime}(\eta)=F_{n}\left(\eta_{n}\right)$ and $F^{\prime}(\mu)=E^{\prime}(\mu)$ whenever $\mu_{m} \neq \eta_{m}$ for any $m=1 \ldots k$. Finally $\operatorname{dim} F \leqq 2 v$ because each Jordan chain for $\left.B_{m}\right|_{F}$ starts in $N$ and has length at most two.

One can specify $\operatorname{dim} F$ precisely in terms of the behaviour of $(,)_{0}$ on $N$. Let $M_{0}$ be the (Gram) matrix with $(m, n)$ th element $\left(u^{m}, u^{m}\right)_{0}$ for a suitable basis $u^{1}, \ldots, u^{v}$ of $N$. Appealing to the canonical form ([12], Theorem I.3.3) for $B_{m}^{\prime}$ on $N$, in the (, $)_{m}^{\prime}$ inner 
product, we see that there is a Jordan basis for $B_{m}^{\prime}$ relative to which $M_{0}$ is diagonal with nonzero (resp. zero) elements corresponding to $1 \times 1$ (resp. $2 \times 2$ ) Jordan blocks for $B_{m}^{\prime}$. Thus

$$
\operatorname{dim} F=v+v_{0}
$$

where $v_{0}$ denotes the number of ztro eigenvalues of $M_{0}$. On the other hand, we note that decomposable bases of $N$ also exist ([2], Theorem 4.3.1) and the elements of $M_{0}$ are then determinants with elements of the form $\left(x_{j}, V_{j l} x_{j}\right)$. By the law of inertia ([17], $\S 92$ ), we can determine $v_{0}$, and hence $\operatorname{dim} F$, by means of these simpler matrices, expressible directly in terms of the original data $\left({ }^{*}\right)$.

Finally, let us illustrate some of the above with a special case.

Example 5.5. Suppose the eigenspace corresponding to the minimal eigenvalue of each $T_{m}$ has dimension one. In particular, this is the case for the Sturm-Liouville case (1.1) with separated end conditions-cf. [9] for $k=2$.

Three cases can arise. First, if $T_{m}$ is positive definite for some $m$, then $v=0$ and so $\operatorname{dim} F=0$. The eigentensors are complete in $H$, and we have the situation of LD-in fact the problem is transformable to one satisfying LD ([3], Corollary 2.2 and subsequent references).

Assume now that $T_{m} x_{m}=0$ for some nonzero $x_{m} \in H_{m}, m=1, \ldots, k$. Then $N$ is spanned by $x^{\otimes}=x_{1} \otimes \cdots \otimes x_{k}$. Evidently $v=1$, so $\operatorname{dim} F$ depends on $v_{0}$. We note that $\left(x^{\otimes}, x^{\otimes}\right)_{0}$ is a determinant $\delta_{0}$ with $(m, n)$ th entry $\left(x_{m}, V_{m n} x_{m}\right)$. If $\delta_{0} \neq 0$ then $v_{0}=0$, so $\operatorname{dim} F=1$, i.e. $F=N$ and again the eigentensors are complete.

The third case arises when $\delta_{0}=0$, and then $v_{0}=1$, so $\operatorname{dim} F=2$ and the eigentensors are not complete. $F$ is spanned by $x^{\otimes}$ and a common root vector $y$, say, for the $B_{m}^{\prime}$.

Acknowledgements. The research of the first author was supported by NSERC of Canada, and the research of the second author was supported by a Post Doctoral Fellowship at the University of Calgary.

\section{REFERENCES}

1. F. M. Arscott, Periodic Differential Equations: An Introduction to Mathieu, Láme and Allied Functions (Macmillan Press, 1964).

2. F. V. Atxınson, Multiparameter Eigenvalue Problems, Vol. 1 (Academic Press, 1972).

3. P. A. Binding, Multiparameter definiteness conditions, Proc. Roy. Soc. Edinburgh 89A (1981), 319-332.

4. P. A. Binding, Left definite multiparameter eigenvalue problems, Trans. Amer. Math. Soc. 272 (1982), 475-486.

5. P. A. Binding and P. J. Browne, $A$ variational approach to multiparameter eigenvalue problems in Hilbert space, SIAM J. Math. Anal. 9 (1978), $1054-1067$. 
6. P. A. Binding and K. Seddighi, On root vectors of self-adjoint pencils, J. Funct. Anal., to appear.

7. J. Bognár, Indefinite Inner Product Spaces (Springer-Verlag, 1974).

8. K. Daho and H. Langer, Sturm-Liouville operators with an indefinite weight function, Proc. Roy. Soc. Edinburgh 78A (1977), 161-191.

9. M. Faierman, Expansions in eigenfunctions of a two-parameter system of differential equations I-IV, to appear.

10. J. Fleckinger and A. B. Mingarelli, On the eigenvalues of non-definite elliptic operators, Differential Equations (ed. I. W. Knowles, R. T. Lewis) (North-Holland, 1984), 219-227.

11. F. R. Gantmacher, The Theory of Matrices, Vol. 2 (Chelsea, 1959).

12. I. Gohberg, P. LANcaster and L. Rodman, Matrices and Indefinite Scalar Products (Birkhäuser-Verlag, 1983).

13. W. M. Greenlee, Double unconditional bases associated with a quadratic characteristic parameter problem, J. Funct. Anal. 15 (1974), 306-339.

14. D. Hilbert, Grundzüge Einer Allgemeinen Theorie der Linearen Integralgleichungen, 1912 (reprinted by Chelsea, 1953).

15. A. Källstróm and B. D. Sleeman, A left definite multiparameter eigenvalue problem in ordinary differential equations, Proc. Roy. Soc. Edinburgh 74A (1976), 145-155.

16. T. Kato, Perturbation Theory for Linear Operators (Springer-Verlag, 1966).

17. A. I. MAL'Cev, Foundations of Linear Algebra (Freeman, 1963).

18. G. F. Roach and B. D. Sleeman, On the spectral theory of operator bundles II, Applicable Anal. 9 (1979), 29-36.

19. B. D. Sleeman, Multiparameter Spectral Theory in Hilbert Space (Pitman, 1978).

20. H. Volkmer, On multiparameter theory, J. Math. Anal. Appl. 86 (1982), 44-53.

21. H. Volkmer, On the completeness of eigenvectors of right definite multiparameter problems, Proc. Roy. Soc. Edinburgh 96A (1984), 69-78.

Department of Mathematics and Statistics

UNIVERSITY OF CALGARY

Calgary, Alberta

Canada T2N IN4
Department of Mathematics

UNIVERSITY OF SHIRAZ

IRAN 